\title{
Empoderamiento e Inclusión Social de Mujeres Inmigrantes a través de las Tertulias Literarias Dialógicas
}

\section{Empowerment and Social Inclusion of Migrant Women through Dialogic Literary Gatherings}

\author{
Carme García-Yeste * \\ Regina Gairal \\ Oriol Ríos
}

Universitat Rovira i Virgili

\begin{abstract}
La superación de las desigualdades de género es uno de los principales retos de organismos internacionales como la Comisión Europea. En el marco de los objetivos globales de las Naciones Unidas para superar la pobreza y conseguir la prosperidad para todos los colectivos se plantean una serie de directrices dirigidas a avanzar en la mejora de la situación de la mujer. Si bien dichas estrategias visibilizan la preocupación de los principales organismos internacionales y gobiernos alrededor de las injusticias de género, persisten aún problemáticas sobre las que profundizar. El presente artículo analiza cómo las tertulias literarias dialógicas están permitiendo empoderar a las mujeres inmigrantes y disminuir su situación de vulnerabilidad. Se ha desarrollado a partir de un estudio cualitativo con orientación comunicativa, en concreto se han realizado ocho entrevistas semiestructuradas, un grupo de discusión y observaciones comunicativas articuladas en sesiones semanales durante dos cursos escolares (2014-2015 y 20152016). Los resultados muestran que esta actuación educativa de éxito está contribuyendo a mejorar el aprendizaje del idioma, dotando de seguridad a las mujeres, fortaleciendo su auto-estima y reforzando sus relaciones familiares y de amistad.
\end{abstract}

Descriptores: Mujer, Grupo étnico, Comunicación, Lengua, Inmigrante.

Overcoming gender inequalities is one of the main challenges of international organizations such as the European Commission. Within the framework of the United Nations' overall objectives for overcoming poverty and achieving prosperity for all groups, a series of guidelines are proposed to advance the improvement of the situation of women. Although these strategies raise the concern of the main international organizations and governments around gender injustices, there are still problems to be studied. This article analyzes how literary dialogues are enabling immigrant women to be empowered and to reduce their vulnerability. It has been developed from a qualitative study with a communicative orientation, in particular eight semi-structured interviews, a discussion group and communicative observations articulated in weekly sessions during two school years (2014- 2015 and 2015-2016). The results show that this successful educational action is contributing to improve language learning, providing women of selfconfidence, strengthening their self-esteem and their family and friendship relationships.

Keywords: Women, Ethnic groups, Communication, Languages, Inmigrants.

*Contacto: carme.garciay@urv.cat

ISSN: 2254-3139

www.rinace.net/riejs/

revistas.uam.es/riejs
Recibido: $\quad 15$ de abril 2017

$1^{\text {a }}$ Evaluación: 7 de junio 2017

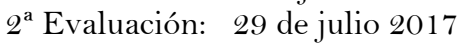

Aceptado: $\quad 26$ de agosto 2017 


\section{Introducción}

La superación de las desigualdades por razón de género es uno de los principales retos que las Naciones Unidas y la Comisión Europea plantea para los próximos años de cara a conseguir mayor justicia e equidad social (Comisión Europea, 2015; ONU, 2015). En este sentido, en el marco de los objetivos globales de las Naciones Unidas para superar la pobreza y conseguir la prosperidad para todos los colectivos (Naciones Unidas, 2015), se plantean una serie de directrices dirigidas a avanzar en la mejora de la situación de la mujer. Por ejemplo, cabe destacar aquellas acciones diseñadas a empoderar a las mujeres y a eliminar las desigualdades de género en educación, sobre todo en el acceso de las mujeres y las niñas a todos los niveles educativos. En esta misma línea, la Comisión Europea publicaba en el año 2015 una estrategia integral para la igualdad de género para el periodo 2016-2019 con la que también proponía como acción central trabajar para la igualdad en el ámbito educativo, sobre todo en la elección de las carreras universitarias.

Si bien dichas estrategias visibilizan la preocupación de los principales organismos internacionales y gobiernos alrededor de las injusticias de género, persisten aún problemáticas sobre las que profundizar. Por ejemplo, la situación de las mujeres inmigrantes, refugiadas o sin titulación académica en algunos países de Europa resulta preocupante. Según datos de la Organización para la Cooperación y el Desarrollo Económico, en el año 2015 el rendimiento escolar de la población inmigrante en la mayoría de países de la OCDE es inferior al de la población nativa. Además, dichos datos correlacionan directamente con la inserción laboral de esta población, que en el caso de las mujeres inmigrantes presenta evidencias de una importante exclusión social. De hecho, en la mayoría de países la tasa de desempleo de las mujeres inmigrantes es superior a la de las mujeres nativas. Cabe subrayar que resulta alarmante la situación en determinados países como España y Grecia donde el desempleo de las mujeres no nativas supera el 30\% (OCDE, 2017). Paralelamente, otra problemática ligada directamente a esta situación de desigualdad hace referencia a los índices de alfabetización de las mujeres. A pesar de los esfuerzos y de los avances conseguidos en este ámbito, según el informe más reciente de UNESCO que analiza dicho fenómeno, se observan unos índices superiores de analfabetismo en el caso de la mujer (UNESCO, 2017).

La investigación centrada en analizar las desigualdades de género hace décadas que ha acuñado el concepto interseccionalidad como forma de expresar la incidencia que tienen las diferentes variables para agravar la situación de vulnerabilidad de las mujeres (Anthias 2013; García-Yeste, 2014; Veenstra, 2013; Yousaf y Purkayastha, 2015). De esta forma este enfoque ha sido de utilidad para profundizar en los efectos que tienen variables como la clase social o el origen étnico en la situación de exclusión de las mujeres. Otro enfoque teórico que ha proporcionado elementos de análisis muy importantes en este ámbito es el feminismo dialógico (De Botton, Puigvert y Sánchez, 2005; Puigvert, 2001). El feminismo dialógico enfatiza la necesidad de dar voz a aquellas mujeres que tradicionalmente han sido silenciadas de las luchas feministas a favor de la igualdad. Voces de otras mujeres, trabajadoras domésticas, amas de casa, inmigrantes, gitanas que están liderando cambios en sus vidas personales y en las de su comunidad para terminar con las injusticias sociales. 
De este modo, en el presente artículo partiremos de ambos enfoques para describir las transformaciones que un grupo de mujeres inmigrantes de la localidad de Tarragona están desarrollando gracias a su participación en una actuación educativa de éxito, concretamente las tertulias literarias dialógicas (TLD) (Flecha, 2015ab). Con este objetivo el artículo concreta cuatro partes diferentes. En la primera se desarrolla una fundamentación teórica donde se abordan algunas de las principales aportaciones alrededor de la interseccionalidad y el feminismo dialógico, en la segunda se describe el método de investigación y la perspectiva metodológica de la que se parte. En el tercer apartado se definen los principales resultados alcanzados a través de la investigación desarrollada y por último se presenta la discusión donde se sintetizan las principales conclusiones del artículo.

\section{Fundamentación teórica}

La desigualdad de género es una problemática ampliamente estudiada desde hace décadas por la investigación social. En este apartado vamos a profundizar sobre ello considerando enfoques teóricos diversos tales como la interseccionalidad, el feminismo dialógico y el análisis postcolonial. Además, vamos a presentar algunas de las características que definen las actuaciones educativas de éxito, una aproximación que profundiza en el impacto que tienen determinadas prácticas para mejorar la vida de los colectivos más favorecidos, entre los que están las mujeres inmigrantes.

La interseccionalidad es un término que fue desarrollado por Crenshaw (1991) con el objetivo de ofrecer explicaciones mutidimensionales al fenómeno de la desigualdad y la injusticia social. Para la autora resultaba crucial analizar la definición de las identidades sociales considerando la interrelación entre los diferentes elementos de opresión, dominación y discriminación que dificultan su desarrollo. Precisamente, Crenshaw argumenta que existen determinados factores culturales y biológicos que unidamente pueden explicar la reproducción de los mecanismos que llevan a los sujetos a la exclusión social, como son el género, la etnia, la clase social, la orientación sexual, la edad, la nacionalidad, etc.

Los análisis recientes que han utilizado esta aproximación teórica para dotar de explicaciones rigurosas al porqué de las injusticias sociales, ofrecen diferentes argumentos a tener en cuenta. Por ejemplo, algunos estudios señalan que el racismo y el sexismo están estrechamente ligados ya que las dimensiones de género y etnia son claves para configurar la personalidad de los sujetos. Ambas variables ayudan a categorizar a las personas y establecer prácticas de discriminación que refuerzan los estereotipos racistas y sexistas (Gianettoni y Roux, 2010). Estos análisis tienen su punto de partida en los estudios culturales y en la race critical theory en los que se pone de manifiesto que la intersección de la variable género, clase social y etnia es determinante para comprender las injusticias que sufren colectivos como las mujeres afro-americanas (James, 2012). Estos análisis ofrecen un cuerpo argumentativo importante para profundizar alrededor de las desigualdades de género, pero no proporcionan qué estrategias y prácticas han permitido superarlas. En las siguientes líneas se van a describir brevemente algunas de estas estrategias y planteamientos aportados por el feminismo dialógico.

La segunda ola del feminismo nació en el marco de una modernidad que empezaba a cuestionar algunas reivindicaciones que había liderado el feminismo de la igualdad 
(Beck-Gernsheim, Butler y Puigvert, 2003). Éste último planteaba como objetivo principal conseguir la equidad respecto al hombre, aunque para conseguirlo se construyera un movimiento social basado en la homogeneidad. En otras palabras, el feminismo de la igualdad no cuidó que la diversidad de identidades femeninas se incluyera en sus luchas, de modo que el feminismo de la diferencia más tarde cogió por bandera el derecho a ser diferente como eje central de sus reivindicaciones (Irigaray, 1998). Así entre sus posturas destacaba la importancia de la diferenciación-oposición con el hombre como elemento que definía la construcción de la mujer: "Nacer mujer, antes de significar ser una generadora de la humanidad, comporta el sentido de encarnar el ser mujer con el otro-hombre, con el ser hombre" (p. 45).

Una de las carencias presentes en las posturas anteriormente presentadas se vincula a la invisibilidad de las otras mujeres en las reivindicaciones feministas. Las otras mujeres son aquellas mujeres que tradicionalmente no han participado en el movimiento feminista, que también han sido excluidas de los organismos públicos y lugares de responsabilidad de la sociedad. Se trata de mujeres inmigrantes, de minorías culturales, mujeres sin titulación académica que con frecuencia se encuentran en situación de exclusión social (García, Larena y Miró, 2012). La literatura científica ha nombrado las reivindicaciones y conquistas de estas mujeres como feminismo dialógico (Christou y Puigvert, 2011). Una de las reivindicaciones en los que han trabajado con mayor esfuerzo las otras mujeres es en la transformación de la educación. En este sentido, cabe subrayar que para conseguir esta transformación las otras mujeres empiezan a participar en oportunidades formativas que mejoren su situación. Así, muchas de ellas inician procesos de alfabetización o cursan cursos para obtener el grado de educación secundaria o acceder a la universidad en centros de educación de personas adultas o formación permanente (Oliver et al., 2016).

Sin embargo, el interés en el mundo educativo va más allá de la propia formación de las mujeres, de hecho, una de las realidades en las que están haciendo mayor hincapié el feminismo dialógico es en la participación de las familias y la comunidad en la escuela para apoyar la educación de sus hijos, hijas, nietos y nietas (Christou y Puigvert, 2011; Beck-Gernsheim, Butler y Puigvert, 2003). De este modo las mujeres reivindican implicarse en actuaciones que tengan un efecto en la vida de los menores, es decir que mejoren su aprendizaje y la convivencia en los centros escolares (Oliver, Soler y Flecha, 2009). En este sentido, ponen especial énfasis en poder tomar parte en las decisiones relevantes de los centros educativos, en actuaciones que aceleren el rendimiento académico del alumnado y que prevengan la violencia en las aulas (Redondo-Sama, 2016). Asimismo, se constata a través de estos análisis que la educación se visualiza como la herramienta de transformación que las otras mujeres consideran central para transformar su vida y la de sus familias.

En este marco de modernidad reflexiva y cambios en los movimientos sociales globales como por ejemplo el feminismo, Beck, Giddens y Lash (1994) destacan las consecuencias que conllevan estas transformaciones en la vida de las mujeres, como por ejemplo su mayor visibilidad en los espacios de decisión pública. En el marco de estos discursos ligados a la modernidad se articulan otros posicionamientos que hacen referencia al pensamiento feminista postcolonial, dando especial importancia a las corrientes y los planteamientos de la descolonización. En este sentido, desde el punto de partida de las ideas proporcionadas por el postcolonialismo, Marchand (2009) también destaca que es 
necesario dar voz y espacios de participación a las mujeres silenciadas, sobre todo las pertenecientes a colectivos vulnerables como las minorías étnicas o religiosas.

Esta participación, sobretodo la ligada al ámbito educativo, es uno de los aspectos que abordan las tertulias literarias dialógicas, actuación en la que intervienen las mujeres protagonistas de esta investigación (Álvarez et al., 2016). Las tertulias literarias dialógicas se caracterizan por la lectura compartida de clásicos de la literatura universal. Concretamente son una actuación que se define a través del diálogo igualitario que se establece entre un grupo de personas que leen y reflexionan alrededor de un libro con el objetivo final de fomentar la creación conjunta de conocimiento (Soler, 2015a). Los libros y los textos que se escogen para la lectura son consensuados entre las participantes y la coordinadora de la tertulia, la cual se encarga de estructurar y dirigir la discusión a través de turnos de palabra. Dicha actuación está consiguiendo un importante impacto (Flecha, Soler y Sordé, 2015), sobre todo por lo que respecta a la mejora de las competencias lectoras y a la educación en sentimientos (Llopis et al., 2016). El proyecto integrado INCLUD-ED. Strategies for inclusion and social cohesion from education in Europe (2006-2011), financiado por el sexto Programa Marco de la Comisión Europea, analizó dicha experiencia a la que consideró una actuación educativa de éxito porque independientemente donde se aplique consigue los mejores resultados (Flecha y Soler, 2014).

Como veremos en el apartado de resultados, las mujeres inmigrantes y de minorías étnicas que están formando parte de las tertulias literarias dialógicas están dando grandes pasos para conseguir una mayor justicia social. Están en la línea del objetivo que la Comisión Europea se plantea para mejorar la educación en la Unión Europea: por un lado, favorecer la igualdad desde las políticas educativas, y por el otro, conseguir la efectividad de los sistemas educativos de forma que los máximos aprendizajes sean una finalidad central. Los últimos avances en el campo de la superación de la pobreza ya indican que es necesario aplicar este tipo de estrategias de éxito, basadas en evidencias, para conseguir unas políticas y prácticas sociales y educativas que tengan un impacto social relevante (Ruiz-Eugenio, 2016). Así pues, como evidenciaremos a continuación, las tertulias literarias dialógicas son un buen ejemplo de todo ello, porque permiten plantear, como señalaría Freire (1993), en una pedagogía de la esperanza para un mundo mejor.

\section{Método}

Partiendo del paradigma comunicativo la investigación que sustenta este artículo es la metodología comunicativa (Gómez, Puigvert y Flecha, 2011). Esta metodología ha sido reconocida por la Comisión Europea como la mejor herramienta para el análisis de la situación de los grupos vulnerables, como las mujeres inmigrantes (Comisión Europea, 2010). Además, diferentes investigaciones del Programa Marco Europeo han utilizado dicho enfoque para analizar diferentes realidades como la inclusión de la comunidad gitana en el mercado laboral, las actuaciones educativas que consiguen mayor cohesión social y éxito educativo, y las formas de solidaridad que mejoran la realidad social (Flecha, 2006; Flecha, 2001; Soler, 2015b).

Una de las características más importantes que definen la metodología comunicativa es la ruptura de la jerarquía interpretativa. Esta premisa significa que las personas investigadoras e investigadas construyen el conocimiento conjuntamente a través de sus 
aportaciones. De este modo, el objetivo es llegar a un entendimiento y por eso el diálogo igualitario es el eje vertebral de las técnicas comunicativas en las que la persona investigadora aporta su bagaje científico y la persona investigada contribuye con sus experiencias ligadas al mundo de la vida (Gómez et al., 2006). Otra de las características importantes de la metodología comunicativa es la voluntad de transformación social, es decir que el conocimiento que se genera a través de la intersubjetividad tiene como principal propósito contribuir a superar las desigualdades e injusticias sociales.

\subsection{Muestra y técnicas de recogida de información}

El estudio que se expone en el presente artículo es de carácter cualitativo con orientación comunicativa. En concreto se han aplicado tres técnicas de recogida de la información diferentes: entrevistas semiestructuradas, observaciones comunicativas y grupos de discusión. Así se han realizado 8 entrevistas semiestructuradas a las mujeres inmigrantes participantes en las tertulias literarias dialógicas, un grupo de discusión también con dichas mujeres y observaciones comunicativas articuladas en sesiones semanales durante dos cursos escolares (2014-2015; 2015-2016) en las mencionadas tertulias literarias dialógicas. Concretamente, este proceso de recogida de datos pretendía responder al siguiente objetivo: identificar los elementos de empoderamiento, ligados al aprendizaje y a la inclusión social, que tienen las tertulias literarias dialógicas para las mujeres inmigrantes que participan en ellas.

En el cuadro 1 se detalla el desglose de los perfiles de la muestra seleccionada $(\mathrm{N}=8)$. Todas las participantes cumplen seis pautas homogéneas: son mujeres, son musulmanas, están casadas, han nacido en Marruecos, son amas de casa y residen en un barrio de Tarragona. La pauta heterogénea que se destaca sería el nivel académico de cada una de ellas. Mientras que algunas de las participantes únicamente han realizado algunos cursos de primaria, otras han accedido a la universidad, aunque ninguna de ellas ha finalizado dichos estudios. La edad también ha sido un ítem diferenciador, gracias a éste durante las tertulias se han desarrollado debates intergeneracionales. Finalmente destacar que la diferencia de años que llevan residiendo en España se ha destacado ya que en los resultados se ha evidenciado en su fluidez con el idioma.

Cuadro 1. Perfiles de la muestra seleccionada

\begin{tabular}{lclc}
\hline \multicolumn{1}{c}{ NOMBRE } & EDAD & \multicolumn{1}{c}{ NIVEL EDUCATIVO } & AÑOS EN ESPAÑA \\
\hline Haris & 33 & Primer curso de secundaria & 11 años \\
Marwa & 36 & Universidad & 10 años \\
Farida & 38 & Segundo curso de universidad & 13 años \\
Fátima & 26 & Secundaria & 7 años \\
Hasna & 34 & Primaria & 4 años \\
Ghaliya & 27 & Bachillerato & 9 años \\
Asmae & 45 & Primaria & 13 años \\
Maroa & 38 & Primaria & 11 años \\
\hline
\end{tabular}

Nota: Los nombres asignados a los perfiles de la muestra son pseudónimos. En los resultados se ha valorado incluir una cita de una niña que estaba presente mientras se realizaba la entrevista a su madre, esta cita da respuesta al objetivo de investigación del presente artículo. Para poder incluir la cita se ha solicitado el permiso correspondiente a la madre.

Fuente: Elaboración propia.

Subrayar que todas las mujeres participantes en la investigación son madres de un centro de educación primaria de Tarragona, en Cataluña. La escuela es de una línea, cuenta con un total de 74 familias y 127 alumnos/as distribuidos en el segundo ciclo de educación infantil, ciclo inicial, ciclo medio y ciclo superior de primaria. Dicho centro 
está situado en el barrio de Camp-Clar, un barrio que nació entre los años 50 y 60 debido al incremento de población inmigrada de España que acudía a Tarragona en aquel periodo. En la actualidad tiene como principales habitantes familias gitanas y familias inmigrantes, principalmente de Marruecos. La situación socio-económica del barrio es de una importante degradación y un porcentaje importante de su población se encuentra en riesgo de exclusión social (Ajuntament de Tarragona, s. f). Las mujeres participantes en las tertulias literarias dialógicas se encuentran una vez por semana en el centro escolar mencionado para leer un libro de literatura clásica universal. Estos encuentros están gestionados por una voluntaria que ejerce de coordinadora y moderadora de la tertulia.

\subsection{Análisis de los datos}

Para el análisis de la información cualitativa obtenida a través de los instrumentos de recogida de datos se ha tenido en cuenta la orientación comunicativa seguida en el estudio. Todas las técnicas han sido transcritas de modo que los argumentos textuales planteados por las personas entrevistas han sido clasificados considerando dos dimensiones diferentes: dimensión exclusora y dimensión transformadora. La primera dimensión hace referencia a aquellas barreras que las mujeres inmigrantes encuentran en sus vidas para poder superar las desigualdades sociales y educativas; la segunda dimensión tiene en cuenta qué contribuciones están haciendo las tertulias literarias dialógicas para superar dichas barreras y fortalecer el empoderamiento de las mujeres inmigrantes.

\section{Resultados}

En este apartado vamos a presentar algunas citas de las personas que han participado en el trabajo de campo con el objetivo de mostrar diferentes evidencias que responden al objetivo principal de la investigación, el cual se ha presentado en el anterior apartado. Esto significa que para la estructuración de este apartado se ha tenido en consideración exclusivamente aquellos elementos transformadores que están haciendo posible el empoderamiento de las mujeres inmigrantes, en particular a través de su participación en las tertulias literarias dialógicas. De este modo se han diferenciado tres secciones diferentes, 1) Aprendizaje del idioma: el impacto de la participación de las TLD en la adquisición de habilidades comunicativas en español; 2) Empoderamiento y seguridad: la mejora de la autoestima para enfrentarse a situaciones cotidianas que ayudan a una mayor inclusión social; y 3) Amistad y Familia: fortalecimiento de los lazos de amistad y las relaciones familiares, uno de los efectos más importantes derivados de la participación de las mujeres inmigrantes en las TLD.

\subsection{Aprendizaje del idioma}

La tertulia literaria dialógica no se centra exclusivamente en leer un libro y comentarlo, implica muchas más cosas. De modo que para la mayoría de las madres participantes en este espacio ha significado poder profundizar en el vocabulario y en la lengua española. La lectura del texto ha comportado analizar y discutir alrededor del significado de las palabras que se están leyendo y ello se ha traducido en un aprendizaje mucho más completo de la lengua. En la siguiente cita una de las mujeres entrevistadas así nos los señala: 
Si hay solo una palabra que no entendemos, nosotras hablamos mucho acerca de esta palabra, damos vueltas y esto es muy importante. He estado un año, pero como si hubiera estado 5 años. (Haris, 33)

Los efectos que tiene asistir y leer libros clásicos en las mujeres, como por ejemplo La casa de Bernarda Alba, van más allá del propio centro educativo o de las relaciones entre miembros de su comunidad. Gracias a la complejidad que estas obras tienen, el aprendizaje de la lengua española es suficientemente fuerte como para poder utilizarlo en otros contextos importantes de la vida de las mujeres inmigrantes. En la siguiente cita una de las mujeres nos describe como ha superado una barrera cuando acude a los servicios de salud. Previamente a su asistencia a las tertulias se encontraba con muchas dificultades para relacionarse con los profesionales, ahora esta situación ha cambiado y puede explicar muy bien qué le sucede a ella y a sus hijos.

\begin{abstract}
Me apunté para aprender el idioma, para mejorar mi vocabulario. Mi intención cuando me apunté era poder hablar perfectamente, porque cuando iba al hospital tenía muchos problemas para explicarle al doctor lo que me pasaba a mí o a mis hijos. $Y$ ahora ya puedo explicarlo mucho mejor. (Farida, 38)
\end{abstract}

Las TLD también han despertado nuevos intereses en sus participantes, intereses ligados a la formación. Si bien a menudo se suelen realizar afirmaciones poco fundamentadas sobre los intereses y las prácticas de las mujeres inmigrantes, con frecuencia sustentadas por planteamientos racistas postmodernos (Mircea y Sordé, 2011), las entrevistas realizadas a las mujeres participantes en las TLD nos demuestran que existe una motivación para adquirir más conocimiento. De hecho, en la siguiente cita se observa que este conocimiento está ligado a elementos que promueven la inclusión de estas mujeres en su país de acogida:

Gracias a Tertulias tenemos muchas ganes de aprender más idiomas, castellano, pero también catalán e inglés. (Haris, 33)

Este aprendizaje no representa una pérdida de las tradiciones y costumbres culturales de estas mujeres, todo lo contrario, ellas continúan manteniéndolas gracias a adquirir nuevas competencias lingüísticas en español. En las entrevistas ellas manifestaban que gracias a conocer mejor el idioma a través de las TLD ahora pueden leer textos del Corán que están traducidos. De alguna forma, dichos argumentos constatan que la inclusión en el país de acogida no tiene por qué ir reñida con el respeto y fomento de la cultura del país de origen.

Ahora leo unos libros del Corán que están escritos en español, antes no los leía porque no los entendía, pero ahora me siento más segura. (Hasna, 34)

El hecho de aprender el idioma del país en el cual residen, tal como se ve en las citas presentadas, ha comportado que las mujeres participantes puedan tener mayores interacciones en su contexto que antes no podían tener. Por ejemplo, ellas perciben que ahora sí pueden hablar con los familiares de los compañeros y compañeras de clase de sus hijos e hijas mientras los esperan a la salida del colegio. También argumentan que les resulta mucho más fácil poder hablar con los maestros y maestras de sus hijos e hijas.

\title{
3.2. Empoderamiento y seguridad
}

Uno de los aspectos que señalan la gran mayoría de las mujeres participantes en la TLD es que han ganado en seguridad. Como se ha indicado previamente, las mujeres inmigrantes deben enfrentarse a una serie de dificultades cotidianas estrechamente ligadas a su inclusión dentro del país de acogida. Sin embargo, uno de los impactos que consigue esta actuación de éxito es empoderar a las mujeres para que tengan la 
seguridad de hacer frente a todas estas situaciones. De esta forma, su identidad y personalidad se ven reforzadas por aspectos positivos que se van aprendiendo en la TLD, sobre todo por el control de la lengua española tanto a nivel oral como escrito. En las dos siguientes citas se pueden constatar dichos efectos:

Las TLD me han ayudado mucho, me dan mucha seguridad para mí misma, antes estaba un poco tímida, pero las TLD me han ayudado mucho para mi personalidad, ahora estoy más abierta para hablar. Mi problema es que si una frase no está bien no puedo hablar y si está bien sí, pero ahora con TLD hablo mucho, he mejorado mi vocabulario y eso me da seguridad en mi misma. (Haris, 33)

Las Tertulias Literarias me sirven para que yo esté más segura, me sirven también para hablar y escribir mejor, me ha ayudado para muchísimas cosas. (Hasna, 34)

Este empoderamiento y seguridad que van consiguiendo las mujeres participantes en las TLD también se concreta en su autoimagen y en la que proyectan a la sociedad. En la siguiente cita podemos observar como una de las mujeres entrevistadas señala la capacidad que pueden tener las mujeres para compaginar su vida personal y familiar con la profesional o laboral. De alguna manera los debates que se tienen a través de las lecturas de obras clásicas abren la puerta a hacer reflexiones sobre el papel de la mujer y ello repercute en la concepción que las participantes en la TLD tienen alrededor de los roles de género y la igualdad (García-Yeste, 2014; Flecha, 2015ba).

[...]Las mujeres podemos hacer muchas coses, trabajar, llevar las cosas de la casa, cuidar de los niños... (Marwa, 36)

Además, otra de las realidades que constatamos en las entrevistas es que este empoderamiento y seguridad hace visible el posicionamiento de las mujeres inmigrantes alrededor de su vida y su identidad. Como veremos en las siguientes citas, ellas consiguen desmitificar algunos de los planteamientos que existen alrededor de la situación de la mujer árabe. Por ejemplo, rompen con la idea que son mujeres dependientes que no quieran estudiar o trabajar:

\section{[...]Tenemos que estudiar para no depender de nuestros maridos. (Fátima, 26)}

También cuestionan que algunas de sus opciones de vida ligadas a la forma de vestir y actuar vengan derivadas al inmovilismo. Por ejemplo, una de las mujeres afirmaba rotundamente que la decisión de llevar hiyab no significa que tengan menos capacidad de reflexión, decisión y emancipación:

\section{[…]Nos tapamos nuestro cuerpo, pero no nuestra mente. (Ghaliya, 27)}

Estas citas evidencian como las mujeres al participar en las TLD rompen estereotipos dejando atrás la visión que algunos grupos de personas tienen acerca de la mujer musulmana, como por ejemplo que son mujeres pasivas e incultas. Al participar en las TLD y con las interacciones que se promueven en esta actuación educativa de éxito, se muestra que son mujeres con ganas de aprender el idioma para poder interactuar con su entorno y formarse. Gracias a participar de estos espacios interculturales y leer obras de la literatura española las mujeres protagonistas de este artículo están demostrando que la formación de calidad es una herramienta de transformación personal y social muy importante.

\subsection{Amistad y familia}

Otros de los aspectos transformadores que las TLD han comportado en la vida de las mujeres están vinculados a la amistad y la familia. En este sentido, gracias a los análisis a los que se llega a través de la lectura de libros de la literatura clásica universal donde se 
aborda la amistad femenina o las relaciones sentimentales, como La casa de Bernarda Alba o Bodas de Sangre, se van articulando unas dinámicas que promueven mejoras significativas. Por ejemplo, las TLD han generado lazos de amistad más allá del momento específico en el que las mujeres se encuentran en el centro escolar (Duque y Teixido, 2016). De hecho, ha generado nuevos momentos y espacios de amistad con el que compartir la vida generando así nuevos incentivos para tener una vida social saludable y enriquecedora. Por ejemplo, al finalizar el curso académico y antes de las vacaciones de verano, las propias participantes organizan una fiesta de final de curso donde se reúnen todas. Al compartir experiencias personales durante el transcurso de las TLD hace que empaticen mucho con la situación de soledad con la que se encuentran en España al tener la familia lejos. Los testimonios de las mujeres recogidos en este apartado evidencian estos hechos.

Con Farida hablamos muchas veces, salimos al parque juntas con los niños. Ahora me he comprado un coche y como ella también tiene uno salimos juntas al parque de Tarragona o a la playa... Antes no nos conocíamos, pero cuando yo me apunte a TLD ahora conozco a muchas mujeres. (Maroa, 38)

Por lo que respecta a la familia, las TLD han fortalecido algunas prácticas y dinámicas que las mujeres mantenían en el seno de sus hogares con sus hijos, hijas y maridos. En este sentido, estos últimos observan como sus madres y parejas desde que participan en las TLD han mejorado su expresión oral y han adquirido nuevos conocimientos y la escuela les dota de un mayor protagonismo y valoración por todo ello. En la siguiente cita, efectuada por la hija de una de las participantes en el trabajo de campo, se da cuenta de ello:

\section{To le apoyo, yo quiero que aprenda, de esta manera será más inteligente. Ahora ya es inteligente, pero si sabe un idioma más, lo será aún más.}

De forma parecida a los cambios en las interacciones con los hijos e hijas, también con los compañeros y maridos existen transformaciones en las interacciones gracias a la participación en las TLD. Al tratarse de un espacio en el que se comparten sentimientos, una de las mujeres manifestaba en una de las entrevistas que ahora tiene conversaciones con su marido en la que profundizan mucho más, en las que ella se siente más cómoda para expresarle emociones que están muy ligadas a las lecturas de los libros. En la siguiente cita podemos constatar este cambio.

Es verdad, hablaré con mi marido y le explicaré que a mí no me da vergüenza que él llore o que me explique cómo está, sino que me gustaría que lo hiciera. (Asmae, $45)$

Los testimonios de las mujeres en el presente estudio nos enseñan la existencia de mecanismos empoderadores de las TLD. Mecanismos que están ayudando a que mujeres con limitaciones y barreras en su vida cotidiana encuentren vías de superación. Sus palabras muestran que esta mejora ha ido de la mano de articular espacios e interacciones donde la formación y la reflexión han sido dos elementos centrales.

\section{Discusión y conclusiones}

Las desigualdades de género muy a menudo se agudizan cuando otras variables interseccionan con ellas, como por ejemplo la nacionalidad, la etnia o la clase social. En este artículo hemos podido ahondar en aquella literatura que analiza en profundidad dicho fenómeno (Brah y Phoenix, 2013; Crenshaw 1991; García-Yeste, 2014; Gianettoni 
y Roux, 2010; James, 2012; McCall, 2005). En este sentido, hemos constatado que la estereotipación de género se duplica cuando la variable etnia también está presente, es decir, los factores de riesgo para encontrarse en una situación de exclusión social se incrementan.

Una parte importante de los estudios feministas se dedican a analizar qué mecanismos permiten superar estas injusticias desde una perspectiva igualitaria, pero a la vez respetando la diversidad cultural (De Botton, Puigvert y Sánchez, 2005; Oliver, Soler y Flecha, 2009). Es de esta forma como el feminismo dialógico señala prácticas efectivas para no reproducir las discriminaciones de género que sufren mujeres de diferentes orígenes étnicos o inmigrantes, partiendo del principio de la igualdad de diferencias (Puigvert, 2001). En este sentido ponen especial hincapié en la necesidad de articular actuaciones educativas de éxito que consigan erradicar la discriminación que sufre la mujer por el hecho de ser mujer e inmigrante, gitana o de cualquier otra etnia (GarcíaYeste, 2014). Entre estas actuaciones se encuentran las tertulias literarias dialógicas que han conseguido, como hemos podido corroborar en los resultados de la investigación presentados, empoderar a mujeres inmigrantes y facilitarles la inclusión social en la sociedad de acogida además de romper con determinados estereotipos de género que normalmente giran a su alrededor. Además, los diálogos y las distintas interacciones que han tenido las mujeres participantes en las TLD han contribuido también a generar nuevas relaciones de amistad entre las participantes y un interés por la cultura del país de acogida.

Las contribuciones sobre formación docente a colectivos minoritarios, como los efectuados por Ball (2006), señalan la importancia de educar desde el fomento de la ciudadanía crítica potenciando al mismo tiempo la implementación de prácticas escolares que vayan destinadas al cambio social. Estos planteamientos en el ámbito de la educación, en la línea de los descritos a lo largo del artículo, son cruciales para construir una mayor justicia social y compensar a aquellos grupos vulnerables que históricamente han sido silenciados o excluidos. Ladson-Billing y Tate (1995) ponen también el acento en este aspecto e insisten en la importancia de incluir las voces de estos colectivos para conseguir unos sistemas educativos más justos e igualitarios:

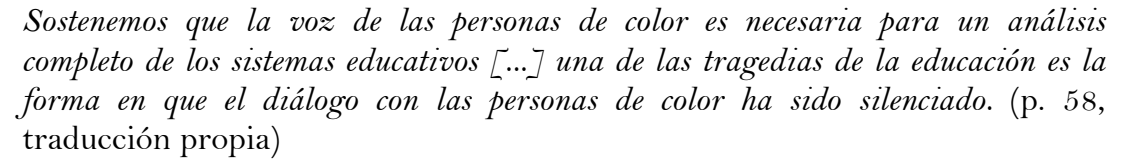

Las TLD se configuran con este objetivo y como hemos podido comprobar obtienen importantes resultados. Por un lado, consiguen mejorar los aprendizajes del idioma en el país de acogida, por el otro lado, y ligado a este último aspecto, las TLD refuerzan la autoestima y la seguridad de las mujeres para hacer frente las barreras cotidianas. Por último, las TLD también mejoran aspectos muy relevantes en los mundos de la vida (Habermas, 1999) de sus participantes, como por ejemplo sus relaciones familiares y los lazos de solidaridad con sus amistades.

\section{Referencias}

Álvarez, P., García-Carrión, R., Puigvert, L., Pulido, C. y Schubert, T. (2016). Beyond the walls. The social reintegration of prisoners through the dialogic reading of classic universal 
literature in prison. International Journal of Offender Therapy and Comparative Criminology, $60(14), 1-19$

Anthias, F. (2013). Moving beyond the janus face of integration and diversity discourses: Towards an intersectional framing. The Sociological Review, 61, 323-343. https://doi.org/10.1111/1467-954X.12001

Ajuntament de Tarragona. (s.f). Pla integral de camp clar. Recuperado de https://www.tarragona.cat/la-ciutat/plans-integrals/pla-integral-de-campclar

Ball, A. F. (2006). Multicultural strategies for education and social change: Carriers of the torch in the United States and South Africa. Nueva York, NY: Teachers College Press.

Beck, U., Giddens, A. y Lash, S. (1994). Reflexive modernization. Politics, tradition and aesthetics in the modern social order. San Francisco, CA: Stanford University Press

Beck-Gernsheim, E., Butler, J. y Puigvert, L. (2003). Women \& social transformation. Nueva York, NY: Peter Lang.

Brah, A. y Phoenix, A. (2013). Ain't IA woman? Revisiting intersectionality. Journal of International Women's Studies, 5(3), 75-86.

Christou, M. y Puigvert, L. (2011). The role of other women in current educational transformations. International Studies in Sociology of Education, 21(1), 77-90. https://doi.org/10.1080/09620214.2011.543855

Comisión Europea. (2010). Conclusions science against poverty. Segovia: MICINN. Recuperado de http://www.idi.mineco.gob.es/stfls/MICINN/Presidencia\%2oEuropea/Ficheros/Confere ncia_Ciencia_contra_la_pobreza.pdf

Comisión Europea. (2015). Strategic engagement for Gender Equality 2016-2019. Luxemburgo: European Commission. Recuperado de http://ec.europa.eu/justice/genderequality/document/files/strategic_engagement_en.pdf

Crenshaw, K. (1991). Mapping the margins: Intersectionality, identity politics, and violence against women of color. Stanford Law Review, 43(6), 1241-1299. https://doi.org/10.2307/1229039

De Botton, L., Puigvert, L. y Sánchez, M. (2005). The inclusion of other women: Breaking the silence through dialogic learning. Nueva York, NY: Springer Science \& Business Media. https://doi.org/10.1007/1-4020-3681-7

Duque, E. y Teixido, J. (2016). Bullying y género. Prevención desde la organización escolar. REMIE. Multidisciplinary Journal of Educational Research, 6(2), 176-204. https://doi.org/10.17583/remie.2016.2108

Flecha, R. (2001). WORKALÓ. The creation of new occupational patterns for cultural minorities. The gypsy case. Bruselas: European Commission.

Flecha, R. (2006). INCLUD-ED. Strategies for inclusion and social cohesion from education in Europe. Bruselas: European Commission.

Flecha, R. (2015a). Successful educational actions for inclusion and social cohesion in Europe. Nueva York, NY: Springer. https://doi.org/10.1007/978-3-319-11176-6

Flecha, A. (2015b). Isabel, from adult learner to community activist. Qualitative Inquiry, 21(10), 865-871. https://doi.org/10.1177/1077800415611693

Flecha, R. y Soler, M. (2014). Communicative methodology: Successful actions and dialogic democracy. Current Sociology, 62(2), 232-242. https://doi.org/10.1177/0011392113515141 
Flecha, R., Soler, M. y Sordé, T. (2015). Social impact: Europe must fund social sciences. Nature, 528(7581), 193. https://doi.org/10.1038/528193d

Freire, P. (1993). Pedagogía de la esperanza: un reencuentro con la pedagogía del oprimido. Madrid: Siglo XXI.

García, C., Larena, R. y Miró, I. (2012). Overcoming gender stereotypes \& improving learning through the participation of the "other women" in schools. Multidisciplinary Journal of Educational Research, 2(1), 37-55.

García-Yeste, C. (2014). Overcoming stereotypes through the other women's communicative daily life stories. Qualitative Inquiry, 20(7), 923-927. https://doi.org/10.1177/1077800414537218

Gianettoni, L. y Roux, P. (2010). Interconnecting race and gender relations: Racism, sexism and the attribution of sexism to the racialized other. Sex Roles, 62, 374-386. https://doi.org/10.1007/s1 1199-010-9755-9

Gómez, A., Puigvert, L. y Flecha, R. (2011). Critical communicative methodology: Informing real social transformation through research. Qualitative Inquiry, 17(3), 235-245. https://doi.org/10.1177/1077800410397802

Gómez, J., Latorre, A., Sánchez, M. y Flecha, R. (2006). Metodología comunicativa crítica. Barcelona: El Roure.

Habermas, J. (1999). Teoría de la acción comunicativa. Racionalidad de la acción y racionalización social. Madrid: Taurus.

Irigaray, L. (1998). Ser dos. Barcelona: Paidós.

James, C. E. (2012). Students "at risk": Stereotypes and the schooling of black boys. Urban Education, 47, 464-494. https://doi.org/10.1177/0042085911429084

Ladson-Billing, G. y Tate, W. E. (1995). Toward a critical race theory of education. Teachers College Record, 97(1), 47-68.

Llopis, A., Villarejo, B., Soler, M. y Álvarez, P. (2016). (Im)politeness and interactions in dialogic literary gatherings. Journal of Pragmatics, $94, \quad 1-11$. https://doi.org/10.1016/j.pragma.2016.01.004

Marchand, M. H. (2009). The future of gender and development after 9/11: Insights from postcolonial feminism and transnationalism. Third World Quarterly, 30(5), 921-935. https://doi.org/10.1080/01436590902959149

McCall, L. (2005). The complexity of intersectionality. Signs: Journal of Women in Culture and Society, 30(3), 1771-1800. https://doi.org/10.1086/426800

Mircea, T. y Sordé, T. (2011). How to turn difficulties into opportunities: Drawing from diversity to promote social cohesion. International Studies in Sociology of Education, 21(1), 49-62. https://doi.org/10.1080/09620214.2011.543852

Oliver, E., Soler, M. y Flecha, R. (2009). Opening schools to all (women): Efforts to overcome gender violence in Spain. British Journal of Sociology of Education, 30(2), 207-218. https://doi.org/10.1080/01425690802700313

Oliver, E., Tellado, I., Yuste, M. y Larena, R. (2016). The history of the democratic adult education movement in Spain. Teachers College Record, $118(4), 1-31$.

OCDE. (2017). Share of international or foreign students enrolled by field of education. París: OCDE.

Puigvert, L. (2001). Las otras mujeres. Barcelona: El Roure. 
Redondo-Sama, G. (2016). Leadership \& community participation: A literature review. International and Multidisciplinary Journal of Social Sciences, 5(1), 71-92. https://doi.org/10.17583/rimcis.2016.1998

Ruiz-Eugenio, L. (2016). Actuaciones integradas para la superación de la pobreza en escocia: madres adolescentes, familias monoparentales y mujeres musulmanas. Multidisciplinary Journal of Educational Research, 6(3), 208-230. https://doi.org/10.17583/remie.2016.2165

Soler, M. (2015a). Biographies of “invisible” people who transform their lives and enhance social transformations through dialogic gatherings. Qualitative Inquiry, 21(10), 839-842. https://doi.org/10.1177/1077800415614032

Soler, M. (2015b). SOLIDUS: Solidarity in European societies: empowerment, social justice and citizenship. Horizon 2020. Bruselas: European Commission.

UNESCO. (2017). Reading the past, writing the future. Fifty years of promoting literacy. París: UNESCO.

ONU. (2015). Suistainable development goals. Ginebra: UNDP.

Veenstra, G. (2013). The gendered nature of discriminatory experiences by race, class, and sexuality: A comparison of intersectionality theory and the subordinate male target hypothesis. Sex Roles, 68, 646-659. https://doi.org/10.1007/s 11 199-012-0243-2

Yousaf, F. N. y Purkayastha, B. (2015). I am only half alive: Organ trafficking in Pakistan amid interlocking oppressions. International Sociology, 30(6), 637-653. https://doi.org/10.1177/0268580915605648

\section{Breve CV de las autoras}

\section{Carmen García-Yeste}

Profesora agregada de la Universidad Rovira i Virgili. Sus líneas de investigación están relacionadas con las Comunidades de Aprendizaje y el estudio de la superación de pobreza desde el ámbito escolar, sobretodo de los grupos más vulnerables como las minorías étnicas y las personas inmigrantes. Ha sido investigadora principal de proyectos del programa Lifelong Learning, del Plan Nacional I+D+i y de FECYT. Ha publicado en diferentes revistes indexadas, destacando diferentes artículos en revistas incluidas en Q1 de JCR como Violence Against Women o Qualitative Inquiry. ORCID ID: 0000-0001-9717-8021. Email: carme.garciay@urv.cat

\section{Regina Gairal}

Educadora social y pedagoga y desde el año 2015 trabaja en el centro residencial de acción educativa Codina que desarrolla su labor asistencial y pedagógica con niños y jóvenes tutelados. Actualmente también es profesora asociada en el departamento de pedagogía de la Universidad Rovira i Virgili. Por otra parte, también destacar su trayectoria investigadora, ya que ha colaborado como técnica en el Congreso Internacional Multidisciplinar de Investigación Educativa desde el año 2016 hasta la actualidad y como técnica del proyecto: Elaboración de materiales para la prevención y apoyar a las víctimas de violencia escolar financiado por el Plan estratégico de convivencia escolar, Centro Nacional de Innovación e Investigación Educativa. Ha sido coordinadora de Tertulias Literarias Dialógicas con mujeres inmigrantes y gitanas. ORCID ID: 0000-0003-0093-7912. Email: regina.gairal@urv.cat 


\section{Oriol Ríos}

Profesor en la Universidad Rovira i Virgili (Tarragona) en calidad de Investigador Juan de la Cierva dentro del grupo de investigación MEDIS- Metodología de la Investigación Educativa con Impacto Social. Su trayectoria investigadora destaca por el trabajo realizado en torno a las nuevas masculinidades, la coeducación y las desigualdades educativas. Ha participado en varios proyectos europeos y de ámbito nacional en estos ámbitos, a destacar su participación en SALEACOM. Overcoming Inequalities in Schools and Leaning Communities: Innovative Education for a New Century (Marie SkłodowskaCurie (RISE) (2015 hasta 2017) - H2020 y en el proyecto I + D + i Impacto de los Actos comunicativas en la construcción de Nuevas Masculinidades (2010-2012). ORCID ID: 0000-0003-3675-9919. Email: joseoriol.rios@urv.cat 